\title{
Incidence and risk factors for neonatal hypoglycaemia in Kerala, India
}

\author{
CK Sasidharan' ${ }^{1}$ E Gokul ${ }^{2}$ and S Sabitha ${ }^{3}$
}

(Index words: High risk neonates, neonatal hypoglycaemia)

\begin{abstract}
Objectives To detect the incidence and risk factors that predicted the occurrence of neonatal hypoglycaemia and to identify neonates who require mandatory blood glucose screening during the first $48 \mathrm{~h}$ of life.
\end{abstract}

Design Hospital based prospective study in a maternity centre in south India.

Setting Labour room, postnatal wards and newborn nursery of the Institute of Maternal and Child Health, Medical College, Kozhikode, India.

Patients Six hundred and four neonates were enrolled in the study by a systematic random sampling method from 1 August to 1 November 2002.

Intervention/Measurement Random blood glucose levels were estimated by the standard glucose oxidaseperoxidase method on two occasions $24 \mathrm{~h}$ apart, during the first 2 days of life. Nineteen clinical characteristics of the mother-baby pair were analysed statistically in relation to the occurrence of hypoglycaemia.

Results The incidence of neonatal hypoglycaemia in the present study group was 41/1000 live births. Eight variables strongly and independently predicted the risk of neonatal hypoglycaemia, at least one being present in $89.1 \%$ of the hypoglycaemic neonates. They included prematurity, low birthweight, maternal diabetes mellitus, delay in initiation of breastfeeding for more than $2 \mathrm{~h}$ postnatally, maternal pre-eclampsia and eclampsia, birth asphyxia, cold stress or hypothermia, and maternal oligohydramnios.

Conclusions Hypoglycaemia was a common problem in apparently normal asymptomatic babies. Apart from the classic 'text book risk factors', maternal oligohydramnios and a breastfeeding delay of more than $2 \mathrm{~h}$ after delivery predicted the risk of neonatal hypoglycaemia in this group. Mandatory blood glucose screening in babies with any one of the above mentioned risk factors serves as an easy and cost effective measure for the prompt identification of this condition.

\section{Introduction}

Neonatal hypoglycaemia is a preventable cause of neurological sequelae. It is extremely important in developing countries where neonatal mortality accounts for $50-60 \%$ of all infant deaths [1]. Hypoglycaemia, combined with other fuel deficiencies that may occur in association with fetal growth retardation, prematurity and birth asphyxia, may contribute to some of these early deaths.
In large maternity units of the developing world, the disproportionately high number of deliveries and limited resources often make institution of early breastfeeding and maintenance of the warm chain difficult. Hypoglycaemia is surprisingly common in apparently full-term healthy neonates of developing countries [2]. Since 'universal blood glucose screening' may be hazardous [3], need is felt in such centres to detect neonates who are at high risk of developing hypoglycaemia for screening. The present study attempted to detect the incidence of neonatal hypoglycaemia and the risk factors that influence its occurrence in a large maternity centre of India. The study also identifies the neonates who require mandatory blood glucose monitoring during the first $48 \mathrm{~h}$ of life.

\section{Materials and methods}

The incidence and associations of hypoglycaemia have not been accurately described previously at the Institute of Maternal and Child Health (IMCH), Kozhikode, south India, where the annual number of deliveries range between 20000 and 25000 , although the hospital promotes early breastfeeding and prevention of neonatal hypothermia.

The present study was conducted at the labour room, postnatal wards and newborn nursery of IMCH. Sample collection commenced on 1 August 2002, after getting approval from the ethical committee of Kozhikode. Enrollment was made by a systematic random sampling method, every fifth neonate entering the parturition register being selected, after obtaining informed consent from the parents. In case of stillbirths or refusal by the parents, the next neonate was enrolled. By 1 November 2002 , a total of 604 neonates were enrolled, representing 3048 deliveries conducted during this period. Neonates detected to have hypoglycaemia served as cases and those with euglycaemia as unmatched controls. Neonates delivered outside IMCH were excluded from the study.

For every baby in the sample population, relevant antenatal, natal and postnatal details were recorded (Table 1). For the purpose of statistical analysis, hypertensive disorders were subdivided into two groups: gestational hypertension throughout the course (uncomplicated), and cases that progressed to pre-eclampsia or eclampsia (complicated).

Neonatal variables assessed included birthweight, length, ponderal index, Apgar at $1 \mathrm{~min}$ and $5 \mathrm{~min}$, presence of major congenital anomalies, and the interval between delivery and first feed. Rectal temperature of each baby

${ }^{1}$ Professor and Head, ${ }^{2,3}$ junior Residents, Division of Neonatology, Department of Paediatrics, Institute of Maternal and Child Health, Medical College, Kozhikode, India.

Correspondence: EG, Tel:00477957718225, e-mail:<goulerumbala@yahoo.com> (Competing interests: none declared). Received 20 May 2004 and revised version accepted 1 November 2004. 
was recorded before blood glucose estimation. Neonates detected to have cold stress or hypothermia were immediately subjected to warming. Gestational age was assessed, both by calculation from the period of amenorrhoea as well as by using the clinical method [4].

Blood glucose estimation was done on two occasions by the standard glucose oxidase-peroxidase method. All babies detected to have hypoglycaemia (blood glucose values less than $2.2 \mathrm{mmol} / \mathrm{L}$ ) were promptly referred to the newborn nursery where they were treated according to the standard nursery protocol.

Blood grouping and Rh typing of the mother-baby pair were performed. Hematocrit estimation was restricted to 228 patients due to technical constraints.

\section{Data analysis}

To test the influence of above mentioned variables on neonatal hypoglycaemia, the test of independence (chisquare test) was applied. All the interpretations were made at $95 \%$ confidence limits. To eliminate the confounding effects of multiple variables on the outcome, the data was further analysed by the logistic regression method. Variables that strongly and independently predicted hypoglycaemia were validated retrospectively with respect to the present data.

\section{Results}

Out of 604 babies screened, 128 were found to have hypoglycaemia. Thus the incidence of neonatal hypoglycaemia at IMCH was $42 / 1000$ live births. A majority of the cases (84) were detected during the first day. Twelve neonates had low blood glucose values throughout the first 2 days, in spite of the treatment. Fifty two neonates constituted the symptomatic group. Table 1 depicts the clinical characteristics of the mothers and infants with the results of chi-square analysis.

Table 2 shows the logistic regression summary of the above clinical characteristics.

Table 1. Clinical characteristics of mothers and infants $(n=604)$

\begin{tabular}{|c|c|c|c|c|}
\hline No. & Clinical characteristics & Number of cases & Hypoglycaemia & $p$ value \\
\hline \multirow[t]{2}{*}{1.} & Intrauterine growth & & & \\
\hline & SGA & 110 & 38 & 0.02 \\
\hline \multirow[t]{4}{*}{2.} & Ponderal index & & & \\
\hline & $\geq 2.5$ & 422 & 82 & \\
\hline & $2-2.49$ & 158 & 42 & $>0.05$ \\
\hline & $<2$ & 24 & 4 & \\
\hline \multirow[t]{3}{*}{3.} & Gestational age (by period of amenorrhoea) & & & \\
\hline & Preterm & 42 & 12 & $>0.05$ \\
\hline & Term & 562 & 116 & \\
\hline \multirow[t]{3}{*}{4.} & Gestational age (by scoring) & & & \\
\hline & Preterm & 44 & 16 & 0.05 \\
\hline & Term & 560 & 112 & \\
\hline \multirow[t]{3}{*}{5.} & Parity & & & \\
\hline & Primigravida & 314 & 56 & $>0.05$ \\
\hline & Multigravida & 290 & 72 & \\
\hline 6. & Hypertensive disorders of pregnancy (uncomplicated) & 52 & 12 & $>0.05$ \\
\hline 7. & Hypertensive disorders of pregnancy (complicated) & 16 & 8 & 0.05 \\
\hline 8. & Gestational diabetes mellitus & 16 & 8 & 0.05 \\
\hline 9. & Meconium stained amniotic fluid & 62 & 10 & $>0.05$ \\
\hline 10. & Documented fetal heart rate abnormalities & 18 & 4 & $>0.05$ \\
\hline 11. & Duration of rupture of membranes $>24 \mathrm{~h}$ & 40 & 10 & $>0.05$ \\
\hline \multirow[t]{5}{*}{12.} & Mode of delivery & & & \\
\hline & Normal vaginal & 420 & 68 & \\
\hline & Vacuum extraction & 34 & 8 & 0.02 \\
\hline & Forceps & 8 & 0 & \\
\hline & Caesarian section & 142 & 48 & \\
\hline 13. & Birth asphyxia & 14 & 8 & 0.02 \\
\hline \multirow[t]{3}{*}{14.} & Time of first feed & & & \\
\hline & $<2$ hours & 142 & 24 & 0.01 \\
\hline & $>2$ hours & 160 & 56 & \\
\hline 15. & Cold stress or hypothermia & 46 & 28 & 0.0001 \\
\hline 16. & Rh incompatibility & 24 & 6 & $>0.05$ \\
\hline 17. & Oligohydramnios & 26 & 16 & 0.001 \\
\hline 18. & Intrapartum glucose infusion & 30 & 16 & 0.01 \\
\hline \multirow[t]{3}{*}{19.} & Hematocrit & & & \\
\hline & $>60 \%$ & 24 & 8 & $>0.05$ \\
\hline & $<60 \%$ & 204 & 120 & \\
\hline
\end{tabular}

LGA-large for gestational age, SGA-small for gestational age. 
Table 2. Logistic regression summary for dependent variable (hypoglycaemia)

$\mathrm{R}=0.91133848$

Adjusted $\mathrm{R}^{2}=0.82591087$

$\mathrm{P}=0.00001 \quad$ Standard error of estimate $=0.10410$

$\overline{\text { Clinical characteristics that predicted } \quad \text { Weightage }}$

the risk of hypoglycaemia

Gestational age (assessed clinically)

0.061391

Birthweight

0.016427

Delay in initiation of breastfeed $>2 \mathrm{~h}$

0.007734

Birth asphyxia

Cold stress or hypothermia

0.040108

0.083409

Oligohydramnios

0.316515

Hypertensive disorders of pregnancy (complicated) 0.105359

Gestational diabetes mellitus

0.10656

\section{Discussion}

Of the total sample population of 604,128 babies had hypoglycaemia during the first $48 \mathrm{~h}$ of life. The incidence of neonatal hypoglycaemia at IMCH was $42 / 1000$ live births during the study period, an observation with reports from similar centres of the developing world [2].

A majority of the neonates $(65.6 \%)$ developed hypoglycaemia during the first day of life. After being abruptly removed from the constant glucose supply via the placenta, the infant is born with a blood glucose concentration that is proportional to that of the mother. During the first several hours of life there is a rapid fall in this level before compensatory mechanisms start. After reaching a nadir during the third hour of life, blood glucose levels normally stabilise at values exceeding $2.2 \mathrm{mmol} / \mathrm{L}$. However, these homeostatic processes may be disturbed in high risk infants [5].

Hyperinsulinism, secondary to islet cell hyperplasia, is the most common cause of persistent hypoglycaemia during early infancy. This condition may be autosomally inherited or secondary to other causes such as maternal diabetes mellitus [6]. The present study has investigated six neonates with persistent hypoglycaemia, but failed to find hyperinsulinism in them.

Analysis of the clinical data on hypoglycaemic neonates showed a clear preponderance of asymptomatic cases. This observation stresses the importance of prompt screening of the high risk neonates, irrespective of the symptoms. Poor feeding was the most common symptom $(59.2 \%)$, followed by lethargy and irritability $(31.2 \%)$. Hypoglycaemic convulsions were last (6.23\%) in our series.

Patterns of intrauterine growth significantly influenced the incidence of neonatal hypoglycaemia in the present study. A $34.5 \%$ of the small for gestational age (SGA) babies developed hypoglycaemia during the first $48 \mathrm{~h}$ of life, as shown in several reports worldwide [7-9]. Large for gestational age (LGA) babies are also in a precarious situation with respect to glucose homeostasis, hyperinsulinism being responsible for both large birthweight and hypoglycaemia [10].
Published reports conflict on the role of ponderal index as a predictor of neonatal hypoglycaemia. An inverse relationship between it and neonatal hypoglycaemia has been proposed [2], but several workers have challenged this. A report from Cameroon [11] in 1991 has suggested mid-arm circumference as a sensitive predictor of neonatal hypoglycaemia. In the present study, ponderal index failed to influence the incidence of neonatal hypoglycaemia.

Gestational age assessment solely based on the period of amenorrhoea is prone to error. It is reliable only if menstrual cycles of the mother are regular and unmodified by maternal illnesses or oral contraceptives. Our study explored the influence of gestational age, calculated using the period of amenorrhoea and clinical assessment. Analysis revealed that clinically assessed gestational age correlated well with the risk of neonatal hypoglycaemia.

Our data did not show any significant difference in the incidence of hypoglycaemia between infants born to primipara and multipara. Proper guidance regarding healthy breastfeeding practices during the antenatal period itself may explain this. In the state of Kerala, well over $70 \%$ of the expectant mothers receive adequate antenatal care through improved health care delivery systems.

Neonates who suffer intrauterine malnutrition secondary to placental insufficiency are prone to develop neonatal hypoglycaemia [12]. In our study, hypertensive disorders of pregnancy were subdivided as uncomplicated and complicated varieties. A significantly higher incidence of neonatal hypoglycaemia was observed among the latter group. The undisputed role of maternal diabetes mellitus in causing neonatal hypoglycaemia [13] was shown in our series as well. Macrosomia, the hallmark of hyperinsulinism was observed in four of these neonates.

Five variables, viz., meconium stained amniotic fluid, fetal heart rate abnormalities, prolonged rupture of membranes, mode of delivery and birth asphyxia were chosen as indicators of perinatal stress in the present study. While the first three failed to influence the incidence of hypoglycaemia, it was shown that a significant proportion $(33.8 \%)$ of babies delivered by caesarian section and more than half of the asphyxiated neonates (57.1\%) suffered hypoglycaemia. There was a clustering of feeding delay and intrapartum glucose infusion in caesarian section babies, both of which are implicated in causing neonatal hypoglycaemia $[14,15]$. The aetiological role of birth asphyxia in hypoglycaemia is often due to the co-existent perinatal stress, hypoxia, acidosis and increased energy expenditure [16].

In our series, a significant proportion of hypoglycaemic neonates were cold stressed or hypothermic at the time of sampling $(\mathrm{p}=0.0001)$. A feeding delay of more than $2 \mathrm{~h}$ postnatally was also significantly associated with abnormally low blood glucose values in the neonate $(p=0.01)$. These two 'modifiable' risk factors have been recognised as causative factors of neonatal hypoglycaemia in Nepal [2] as well. 
$\mathrm{Rh}$ isoimmunisation [17] and high neonatal hematocrit [2] have been suggested as risk factors for neonatal hypoglycaemia by several authors. In our series, $\mathrm{Rh}$ incompatibility did not appear as a significant risk factor in the causation of neonatal hypoglycaemia. This may be due to the decreased incidence of $\mathrm{Rh}$ isoimmunisation in these cases owing to prior use of anti-D immunoglobulin. A high neonatal hematocrit also did not appear to influence the incidence of hypoglycaemia in this series. A strong correlation between oligohydramnios and neonatal hypoglycaemia may be attributed to the prolonged fetal stress, severe intra-uterine growth retardation (IUGR), undetected premature rupture of membranes (PROM) and fetal congenital anomalies that often accompany this condition [18].

When adjusted for potential confounders by the logistic regression method, the influence of the mode of delivery and maternal intrapartum glucose infusion no longer existed (Table 2). Retrospective validation of the results of logistic regression method in the study group revealed that at least one of the above risk factors was present in 114 out of the 128 hypoglycaemic neonates $(89.1 \%)$.

Thus hypoglycaemia occurs in a significant proportion of neonates in large maternity centres of the developing countries. Appropriate and cost effective interventions to deal with the 'modifiable risk factors' such as feeding delay and hypothermia will definitely bring about a significant reduction in the incidence of this preventable insult to normal neurodevelopment. Mandatory blood glucose screening of all neonates with at least one among the eight prominent risk factors (Table 2) serves as an easy and cost effective measure of detection and treatment of this morbidity.

\section{Acknowledgements}

The statistical support provided by Dr. TPM Fareed, Lecturer, Selection grade, Farook College, Kozhikode and Mr. JS Sudheesh, Lecturer in Statistics, Keltron Institute of Information Technology, Kozhikode, is acknowledged with gratitude.

\section{References}

1. Ashworth A, Waterlow JC. Infant mortality in developing countries. Archives of Disease in Childhood 1982; 57: 882-4.

2. Pal DK, Manandhar DS, Rajbhandari S, RB, Land JM, Patel N, et al. Neonatal hypoglycaemia in Nepal 1. Prevalence and risk factors. Archives of Disease in Childhood 2000; 82: F46-51.
3. Haninger NC, Farley CL. Screening for hypoglycaemia in healthy term neonates: Effects on breastfeeding. Journal of Midwifery and Women's Health 2001; 46: 292-301.

4. Capurro H, Konichezky S, Fonesca D, Caldeyro-Barcia R. A simplified method for the diagnosis of gestational age in the newborn infant. Journal of Pediatrics 1978; 93: $120-2$.

5. Fanconi A, Bovet U, Tschumi A, Litschgi M. Blood glucose in the full term neonate in the first hours following birth. Helvetica Paediatrica Acta 1982; 37: 449-56.

6. Wolfsdorf JI. Hyperinsulinemic hypoglycemia of infancy. Journal of Pediatrics 1998; 132: 1-3.

7. Bhat MA, Kumar P, Bhansali A, Majumdar S, Narang A. Hypoglycemia in small for gestational age babies. Indian Journal of Pediatrics 2000; 67: 423-7.

8. Singhal PK, Singh M, Paul VK, Deorari AK. Neonatal hypoglycaemia. Clinical profile and glucose requirements. Indian Pediatrics 1971; 79: 314-24.

9. Haworth JC, Dilling L, Younoszai MK. Relation of bloodglucose to haematocrit, birthweight, and other body measurements in normal and growth-retarded newborn infants. Lancet 1967; 28: 901-5.

10. Berk MA, Mimouni F, Miodovnik M, Hertzberg V, Valuck J. Macrosomia in infants of insulin-dependent diabetic mothers. Pediatrics 1989; 83: 1029-34.

11. Gozal D, Ndombo PK, Ze Minkande J, Kago I, Tetanye E, et al. Anthropometric measurements in a newborn populatioon in west Africa: a reliable and simple tool for the identification of infants at risk for early postnatal morbidity. Journal of Pediatrics 1991; 118: 800-5.

12. Kolvisto M, Jouppila P. Neonatal hypoglycemia and maternal toxemia. Acta Paediatrica Scandinavica 1974; 63: 743-9.

13. Simmons D, Thompson CF, Conroy C. Incidence and risk factors for neonatal hypoglycaemia among women with gestational diabetes mellitus in south Auckland. Diabetic Medicine 2000; 17: 830-4.

14. Cordero L, Landon MB. Infant of diabetic mother. Clinics in Perinatology 1993; 20: 635-48.

15. Reece EA, Homko CJ. Infant of diabetic mother. Seminars in Perinatology 1994; 18: 459-69.

16. Molteno CD, Malan AF, Heese HD. Neonatal complications and conditions associated with asphyxia neonatorum. South African Medical Journal 1974; 48: 2259-62.

17. Raivio K, Osterlund K. Hypoglycemia and hyperinsulinemia associated with erythroblastosis fetalis. Pediatrics 1969; 43: 217-25.

18. Arias F. Practical Guide to High Risk Pregnancy and Delivery. New Delhi: Harcourt India Pvt. Ltd., 1998. 